\title{
Effects of Sesamin on the Fatty Acid Composition of the Liver of Rats Fed N-6 and N-3 Fatty Acid-Rich Diet
}

\author{
Yoko FujIYAma-Fujiwara, Rumi UMEDA-SAwadA, \\ Mine Kuzuyama, and Osamu Igarashi \\ Institute of Environmental Science for Human Life, Ochanomizu University, \\ Bunkyo-ku, Tokyo 112, Japan
}

(Received September 14, 1994)

\begin{abstract}
Summary Sesamin is known as a specific inhibitor of $\Delta 5$-desaturation, the conversion from dihomo- $\gamma$-linolenic acid $(20: 3(n-6))$ to arachidonic acid $(20: 4(n-6))$. In the previous paper, we reported that sesamin inhibited $\Delta 5$-desaturation of $n-6$ fatty acids in rat hepatocytes but not that of $n-3$ fatty acids, from $20: 4(n-3)$ to $20: 5(n-3)$. Then, we studied the effects of sesamin on 45 -desaturation of $n-6$ and $n-3$ fatty acids in vivo. Rats were fed two types of diets containing sesamin $(0.5 \% \mathrm{w} / \mathrm{w})$ for 4 weeks as follows: in experiment 1 (Exp. 1) $\gamma$-linolenic acid-rich diet and in experiment 2 (Exp. 2) $\alpha$-linolenic acid-rich diet. The fatty acid composition of liver lipids was compared to those of control groups without sesamin. In both Exps. 1 and 2, sesamin increased the liver weight and phospholipid contents in liver. In Exp. 2, sesamin increased n-6 fatty acids and decreased $n-3$ fatty acids even though the diet was rich in $n-3$ fatty acids. Sesamin enhanced the composition ratio of $20: 3(n-6)$ in both Exps. 1 and 2. Decrease of 45 -desaturation index of $n-6$ fatty acid, the ratio of $20: 4(n-6) / 20: 3(n-6)$, by the administration of sesamin suggested that sesamin inhibited the $\Delta 5$-desaturation of $n-6$ fatty acids in the liver. On the contrary, the $\Delta 5$-desaturation index of $n-3$ fatty acids, the ratio of $20: 5(n-3)$ to $18: 3(n-3)$, was increased by sesamin administration in the liver of rats fed $\alpha$-linolenic acid-rich diet (Exp. 2). These results suggested that sesamin inhibited the 45 -desaturation of $n-6$ fatty acids but not that of $n-3$ fatty acids in vivo similarly to results from rat hepatocytes. Key Words sesamin, $\Delta 5$-desaturation, $n-6$ fatty acids, $n-3$ fatty acids, polyunsaturated fatty acid metabolism
\end{abstract}

Sesamin, a lignan of sesame seed, is known to be a potent and selective inhibitor of 45-desaturase. In Mortierella alpina, the addition of sesamin in the culture medium increased the dihomo- $\gamma$-linolenic acid content in the produced oil

Abbreviations: PC, phosphatidylcholine; PE, phosphatidylethanolamine; CE, cholesteryl ester; TG, tri-O-acylglycerol. 
(1-3). In a whole animal study, Sugano et al. reported that the addition of sesamin changed the fatty acid composition in liver phospholipids probably by decreasing $\Delta 5$-desaturase activity (4). Shimizu et al. reported the inhibition of $\Delta 5$-desaturase by sesamin in rat liver microsome preparations using $\gamma$-linolenic and dihomo- $\gamma$ linolenic acids as substrates (5). Previously, we reported that sesamin inhibited the $\Delta 5$-desaturation of $n-6$ fatty acids, from $20: 3(n-6)$ to $20: 4(n-6)$, but not that of $n$ 3 fatty acids, from $20: 4(n-3)$ to $20: 5(n-3)$ in cultured rat hepatocytes (6).

It is generally agreed that $n-6$ and $n-3$ fatty acids compete in the same enzyme systems (7-9). The desaturation steps in this pathway are considered given that $n$ 3 series fatty acids are preferable to $n-6$ series fatty acids (10). From these points of view, the competition of the metabolism of both series of PUFAs and the intake balance of both types of fatty acids in diets is still being discussed, but the studies which measured the activities of desaturase were carried out using only $n-6$ fatty acids as substrates.

The present study was conducted to determine whether or not the difference in the inhibitory effect of sesamin between $n-6$ and $n-3$ fatty acids observed in rat hepatocytes is also observed in vivo.

\section{MATERIALS AND METHODS}

Animals and diets. Three-week-old male rats of the Wistar strain (Nippon Clea Ltd., Tokyo, Japan) were used. The animals were housed individually in an air-conditioned room $\left(22-24^{\circ} \mathrm{C}\right.$ with a 12 -h light cycle) and were given experimental diets and water ad libitum for 4 weeks after feeding commercial diet (CE-2, Nippon Clea) for a week. Twenty of the rats were divided into two groups. The control group was fed the diet based on a standard diet (Eisai Co., Tokyo, Japan) containing $10 \%(\mathrm{w} / \mathrm{w})$ of $\gamma$-linolenic acid-rich oil (a product of $M$. alpina culture medium and donated by Idemitsu Petrochemical Co. Ltd., Tokyo, Japan) which contains ca. $25 \%$ of $18: 3(n-6)$. The other group was given the basal diet with $0.5 \%(\mathrm{w} / \mathrm{w})$ of sesamin added (Exp. 1). Sesamin donated from Suntory Ltd. (Osaka, Japan) was a mixture of sesamin and epi-sesamin $(1: 1, \mathbf{w} / \mathbf{w})$. In Exp. 2, the basal diet contained $10 \%$ of the oil mixture (perilla oil: safflower oil $=7: 3, n-3$ / $n-6$ ratio of $2.8: 1$ ), which is rich in $n-3$ fatty acids, was used for comparison with the results of Exp. 1. In Exp. 2, to reduce the higher content of $\alpha$-linolenic acid, safflower oil was mixed with perilla oil. Fatty acid composition of these dietary oils

Table 1. Fatty acid composition ${ }^{1}$ of dietary oils.

\begin{tabular}{lrrrrrc}
\hline & $16: 0$ & $18: 0$ & $18: 1$ & $18: 2$ & $\begin{array}{r}18: 3 \\
(n-6)\end{array}$ & $\begin{array}{r}18: 3 \\
(n-3)\end{array}$ \\
\hline Exp. 1 ( $\gamma$-linolenic acid oil) & 16.8 & 1.9 & 35.3 & 13.5 & 25.4 & \\
Exp. 2 (perilla : safflower $=7: 3)$ & 6.7 & 2.1 & 36.7 & 14.4 & & 40.3 \\
\hline
\end{tabular}

$1 \%$ of total fatty acids. 
is shown in Table 1.

Lipid analyses. Liver lipid was extracted by the method of Folch et al. (11). The concentration of liver lipids was measured with an Iatroscan (Iatron, Co. Ltd., Tokyo, Japan) by thin-layer chromatography (TLC) (12). Lipids extracted were methylated using $\mathrm{HCl}$-methanol by the method mentioned in the previous paper (13). The fatty acid composition was measured by gas-liquid chromatography (GLC; Shimadzu GC-14A) equipped with FID and a Rascot Silar 5CP capillary column $(0.25 \mathrm{~mm} \times 50 \mathrm{~m})$ of which conditions were described in detail in the previous paper (13). The fatty acid composition of phosphatidylcholine (PC) and phosphatidylethanolamine (PE) of the liver was measured after the separation of PC and PE by TLC, respectively (14).

Statistical analysis. The results are presented as the mean \pm SD. The significance of differences of mean values was evaluated by Student's $t$-test.

\section{RESULTS}

\section{Experiment 1}

Table 2 shows the effect of sesamin on the tissue weight. Sesamin did not affect food intake or growth. In the sesamin treatment group of Exps. 1 and 2, the liver

Table 2. Effect of sesamin on tissue weight.

$(\mathrm{g})$

\begin{tabular}{lccccc}
\hline & \multicolumn{2}{c}{ Exp. 1 } & & \multicolumn{2}{c}{ Exp. 2 } \\
\cline { 2 - 3 } \cline { 5 - 6 } & Control & Sesamin & & Control & Sesamin \\
\hline Body weight & $257 \pm 22$ & $249 \pm 23$ & & $230 \pm 7$ & $226 \pm 10$ \\
Liver & $9.6 \pm 0.75$ & $12.5 \pm 5.1^{* *}$ & & $12.2 \pm 0.6$ & $17.3 \pm 1.3^{* *}$ \\
Kidney & $1.8 \pm 0.2$ & $1.9 \pm 0.2$ & & $2.2 \pm 0.1$ & $2.1 \pm 0.1$ \\
Heart & $0.93 \pm 0.07$ & $0.98 \pm 0.08$ & & $1.1 \pm 0.03$ & $1.0 \pm 0.06$ \\
Lung & $1.1 \pm 0.08$ & $1.1 \pm 0.05$ & & $1.2 \pm 0.04$ & $1.1 \pm 0.12$ \\
Brain & $1.3 \pm 0.02$ & $1.3 \pm 0.04$ & & $1.3 \pm 0.04$ & $1.3 \pm 0.04$ \\
\hline
\end{tabular}

Mean \pm SD $(n=6) . * *$ Significant differences from control $(p<0.01)$.

Table 3. Effect of sesamin on liver lipid composition.

\begin{tabular}{lcc} 
& & $(\mu \mathrm{g} / \mathrm{g}$ liver weight $)$ \\
\hline PC & Control & Sesamin \\
PE & $1,006 \pm 141$ & $1,684 \pm 393^{* *}$ \\
TG & $421 \pm 63$ & $745 \pm 210^{*}$ \\
CE & $177 \pm 30$ & $190 \pm 46$ \\
FC & $35.5 \pm 7.1$ & $36.7 \pm 7.4$ \\
\hline
\end{tabular}

Mean \pm SD $(n=6)$. Significant differences from control: ${ }^{*} p<0.05,{ }^{* *} p<0.01$.

Vol. 41, No. 2, 1995 
Table 4. Effect of sesamin on fatty acid composition of liver lipids.

$(\%)$

\begin{tabular}{lcc}
\hline & Control & Sesamin \\
\hline $16: 0$ & $21.5 \pm 0.5$ & $24.7 \pm 3.0^{*}$ \\
$16: 1$ & $2.1 \pm 0.5$ & $1.6 \pm 0.6$ \\
$18: 0$ & $18.0 \pm 0.5$ & $16.1 \pm 3.2$ \\
$18: 1(n-9)$ & $16.8 \pm 1.4$ & $23.6 \pm 4.2^{* * *}$ \\
$18: 2(n-6)$ & $4.2 \pm 0.4$ & $4.1 \pm 1.0$ \\
$18: 3(n-6)$ & $1.1 \pm 0.2$ & $0.7 \pm 0.2^{* * *}$ \\
$20: 3(n-6)$ & $1.1 \pm 0.1$ & $2.0 \pm 0.4^{* * *}$ \\
$20: 4(n-6)$ & $27.9 \pm 0.8$ & $24.1 \pm 3.6^{*}$ \\
$22: 4(n-6)$ & $1.3 \pm 0.3$ & $0.6 \pm 0.07^{* * *}$ \\
$22: 6(n-3)$ & $2.2 \pm 0.3$ & $1.5 \pm 0.3^{* * *}$ \\
$\Delta 5-$ desaturation index & & \\
$20: 4(n-6) / 20: 3(n-6)$ & $24.5 \pm 1.7$ & $11.9 \pm 2.7^{* *}$ \\
\hline
\end{tabular}

Mean \pm SD. Significant differences from control group: ${ }^{*} p<0.05,{ }^{* *} p<0.01,{ }^{* * *} p<$ 0.005 .

Table 5. Effect of sesamin on fatty acid composition of liver PC.

Mean \pm SD. Significant differences from control: ${ }^{*} p<0.05,{ }^{* *} p<0.01,{ }^{* * *} p<0.005$.

weight was significantly higher than the control group, but the weight of the other organs was not influenced. Sesamin significantly increased the concentration of PC and PE in the liver lipids (Exp. 1, Table 3).

In Table 4, the effect of sesamin on the fatty acid composition of whole lipids of rat liver is shown. In the sesamin group, the composition ratio of $18: 1(n-9)$ and $20: 3(n-6)$ were increased significantly while the composition of $18: 0,22: 4(n-6)$ and $22: 6(n-3)$ decreased. Since the 45 -desaturation index, the ratio of $20: 4(n-6)$ 
Table 6. Effect of sesamin on fatty acid composition of liver PE.

\begin{tabular}{lcc} 
& & \\
\hline & Control & Sesamin \\
\hline $16: 0$ & $16.7 \pm 0.8$ & $17.2 \pm 2.7$ \\
$16: 1$ & $0.4 \pm 0.1$ & $0.9 \pm 0.01^{* * *}$ \\
$18: 0$ & $25.0 \pm 1.4$ & $28.2 \pm 2.8^{*}$ \\
$18: 1(n-9)$ & $3.0 \pm 0.4$ & $5.9 \pm 0.9^{* * *}$ \\
$18: 2(n-6)$ & $1.7 \pm 0.1$ & $2.2 \pm 0.4$ \\
$18: 3(n-6)$ & $0.3 \pm 0.02$ & n.d. \\
$20: 3(n-6)$ & $0.7 \pm 0.1$ & $1.2 \pm 0.3^{* * *}$ \\
$20: 4(n-6)$ & $33.0 \pm 1.1$ & $38.1 \pm 1.9^{* * *}$ \\
$22: 4(n-6)$ & $2.7 \pm 0.4$ & $0.9 \pm 0.2^{* * *}$ \\
$22: 5(n-3)$ & $6.4 \pm 1.1$ & $2.2 \pm 1.0^{* * *}$ \\
$22: 6(n-3)$ & $5.0 \pm 0.4$ & $3.6 \pm 1.1^{*}$ \\
$\Delta 5-$ desaturation index & & \\
$20: 4(n-6) / 20: 3(n-6)$ & $45.5 \pm 6.2$ & $30.3 \pm 30.3$ \\
\hline
\end{tabular}

Mean \pm SD. Significant differences from control: ${ }^{*} p<0.05,{ }^{* *} p<0.01,{ }^{* * *} p<0.005$.

to $20: 3(n-6)$, was decreased by sesamin administration, it was suggested that sesamin inhibited $\Delta 5$-desaturation. Tables 5 and 6 indicate the fatty acid composition of PC and PE of the liver phospholipids, respectively. The main unsaturated fatty acid in PC and PE was $20: 4(n-6) . N-3$ fatty acids, $22: 5(n-3)$ and $22: 6(n-3)$, of liver phospholipids were significantly lower in the sesamin group compared with the control group. 18:3(n-3) in PE was found in the control group but not in the sesamin group. Sesamin increased 16:0 and n-6 fatty acids in the PC and PE fractions. The decrease of the $\Delta 5$-desaturation index suggested that sesamin inhibited $\Delta 5$-desaturation in fatty acids in both PC and PE, but to a smaller extent in PE.

The 45-desaturation index of kidney, heart, lung, and serum also showed similar inhibition by sesamin, namely the conversion of $20: 3(n-6)$ to $20: 4(n-6)$ (data not shown).

\section{Experiment 2}

In contrast with the $\gamma$-linolenic acid-rich oil diet (Exp. 1), both series of $n-6$ and $n-3$ fatty acids, including their metabolites, were detected in all tissues of rats fed the $\alpha$-linolenate-rich diet of Exp. 2. Therefore, we can evaluate the effect of sesamin on desaturation of $n-3$ fatty acids as well. Table 7 shows the effect of sesamin on the change in liver fatty acid composition. Sesamin administration increased the percentage of total $n-6$ fatty acids and decreased that of total $n-3$ fatty acids significantly. The same results were observed in other tissues (data not shown). The desaturation index of $n-6$ fatty acids, $20: 4(n-6) / 20: 3(n-6)$, was decreased by sesamin administration. In $n-3$ fatty acids, however, the desaturation index, which was decribed as the ratio of $20: 5(n-3)$ to $18: 3(n-3)$ because $20: 4(n$ - 
Table 7. Effect of sesamin on fatty acid composition of liver.

$(\%)$

\begin{tabular}{lcc} 
& Control & Sesamin \\
\hline $16: 0$ & $22.4 \pm 0.9$ & $27.5 \pm 1.4^{* * * *}$ \\
$16: 1$ & $3.6 \pm 0.5$ & $2.1 \pm 0.3^{* * *}$ \\
$18: 0$ & $14.4 \pm 0.9$ & $13.3 \pm 1.2$ \\
$18: 1(n-9)$ & $25.5 \pm 2.5$ & $29.9 \pm 1.7^{* *}$ \\
$18: 2(n-6)$ & $7.5 \pm 0.4$ & $6.3 \pm 0.4^{* * *}$ \\
$18: 3(n-3)$ & $2.7 \pm 0.6$ & $0.8 \pm 0.3^{* * *}$ \\
$20: 3(n-6)$ & $0.8 \pm 0.04$ & $1.3 \pm 0.1^{* *}$ \\
$20: 4(n-6)$ & $6.2 \pm 0.6$ & $8.2 \pm 1.1^{* * *}$ \\
$20: 5(n-3)$ & $6.4 \pm 1.3$ & $3.3 \pm 0.7^{* * *}$ \\
$22: 5(n-3)$ & $1.5 \pm 0.3$ & $0.9 \pm 0.2^{* * *}$ \\
$22: 6(n-3)$ & $5.6 \pm 0.9$ & $4.5 \pm 0.3$ \\
Total $n-6$ & $14.5 \pm 0.3$ & $15.8 \pm 0.5^{* * *}$ \\
Total $n-3$ & $16.2 \pm 0.8$ & $9.5 \pm 0.4^{* * *}$ \\
$\Delta 5-$ desaturation index & & \\
$20: 4(n-6) / 20: 3(n-6)$ & $7.9 \pm 1.0$ & $6.3 \pm 0.67^{* * *}$ \\
$20: 5(n-3) / 18: 3(n-3)$ & $2.4 \pm 5.1$ & $4.0 \pm 1.3^{* * *}$ \\
\hline
\end{tabular}

Mean \pm SD. Significant differences from control: ${ }^{*} p<0.05$, ${ }^{* *} p<0.01,{ }^{* * *} p<0.005$.

Table 8. Effect of sesamin on fatty acid composition of liver PC.

\begin{tabular}{lcc} 
& & \\
\hline & Control & Sesamin \\
\hline $16: 0$ & $17.6 \pm 1.6$ & $20.5 \pm 4.1$ \\
$16: 1$ & $0.7 \pm 0.2$ & $0.5 \pm 0.1^{* * *}$ \\
$18: 0$ & $27.4 \pm 1.6$ & $25.9 \pm 3.6$ \\
$18: 1(n-9)$ & $5.4 \pm 1.4$ & $4.8 \pm 2.2$ \\
$18: 2(n-6)$ & $7.3 \pm 1.2$ & $5.2 \pm 2.5$ \\
$18: 3(n-3)$ & $0.5 \pm 0.1$ & n.d. \\
$20: 3(n-6)$ & $1.7 \pm 0.2$ & $2.8 \pm 0.3^{*}$ \\
$20: 4(n-6)$ & $13.7 \pm 2.3$ & $18.6 \pm 3.1^{* * *}$ \\
$20: 5(n-3)$ & $11.8 \pm 0.9$ & $6.2 \pm 0.8^{*}$ \\
$22: 5(n-3)$ & $2.6 \pm 0.3$ & $1.9 \pm 0.4^{* * *}$ \\
$22: 6(n-3)$ & $8.5 \pm 1.0$ & $9.3 \pm 2.2$ \\
Total $n-6$ & $22.7 \pm 1.2$ & $26.6 \pm 2.0^{* * *}$ \\
Total $n-3$ & $23.4 \pm 0.6$ & $17.4 \pm 1.2^{* * *}$ \\
$\Delta 5$-desaturation index & & \\
$20: 4(n-6) / 20: 3(n-6)$ & $8.3 \pm 1.1$ & $6.7 \pm 1.1^{*}$ \\
$20: 5(n-3) / 18: 3(n-3)$ & $25.6 \pm 0.26$ & - \\
\hline
\end{tabular}

Mean \pm SD. Significant differences from control: ${ }^{*} p<0.05$, ${ }^{* *} p<0.01,{ }^{* * *} p<0.005$.

3) was not detected in liver, was significantly increased by sesamin. These results suggest that sesamin selectively inhibited $\Delta 5$-desaturation of $n-6$ fatty acids, from $20: 3(n-6)$ to $20: 4(n-6)$, but not that of $n-3$ fatty acids. 
Table 9. Effect of sesamin on fatty acid composition of liver PE.

\begin{tabular}{lcc} 
& Control & Sesamin \\
\hline $16: 0$ & $5.6 \pm 1.3$ & $14.4 \pm 1.3$ \\
$16: 1$ & $1.3 \pm 0.4$ & $0.8 \pm 0.2$ \\
$18: 0$ & $22.9 \pm 1.5$ & $24.1 \pm 1.5$ \\
$18: 1(n-9)$ & $6.2 \pm 1.3$ & $8.2 \pm 2.6$ \\
$18: 2(n-6)$ & $8.3 \pm 3.4$ & $11.7 \pm 0.6^{* *}$ \\
$18: 3(n-3)$ & $0.9 \pm 0.3$ & n.d. \\
$20: 3(n-6)$ & $0.8 \pm 0.3$ & $1.5 \pm 0.2^{* *}$ \\
$20: 4(n-6)$ & $9.7 \pm 1.8$ & $16.2 \pm 2.0^{* *}$ \\
$20: 5(n-3)$ & $10.1 \pm 1.1$ & $5.8 \pm 1.2^{* *}$ \\
$22: 5(n-3)$ & $2.9 \pm 0.5$ & $1.7 \pm 0.4^{* *}$ \\
$22: 6(n-3)$ & $13.9 \pm 1.7$ & $12.4 \pm 0.5$ \\
Total $n-6$ & $18.8 \pm 1.8$ & $29.4 \pm 0.4^{* * *}$ \\
Total $n-3$ & $27.8 \pm 0.9$ & $19.9 \pm 0.7^{* * *}$ \\
$\Delta 5-$ desaturation index & & \\
$20: 4(n-6) / 20: 3(n-6)$ & $13.9 \pm 7.7$ & $10.5 \pm 1.3$ \\
$20: 5(n-3) / 18: 3(n-3)$ & $10.9 \pm 0.9$ & - \\
\hline
\end{tabular}

Mean \pm SD. Significant differences from control: ${ }^{*} p<0.05,{ }^{* *} p<0.01,{ }^{* * *} p<0.005$.

Tables 8 and 9 show the fatty acid composition of liver PC and PE, respectively. Sesamin increased the incorporation of $n-6$ fatty acids into the phospholipid fractions and decreased that of $n-3$ fatty acids. Arachidonic acid $(20: 4(n-6))$ was incorporated into PC rather than PE. Docosahexaenoic acid $(22: 6(n-3))$ was incorporated into PE rather than PC. $\alpha$-Linolenic acid $(18:(n-3))$ was not detected in either PC or PE in the sesamin group. The desaturation index in PC of $n-6$ fatty acids was significantly decreased by sesamin but in PE very little change was observed. These results were similar to Exp. 1. The desaturation index of $n$ 3 fatty acids in phospholipids was not estimated in the absence of $18: 3(n-3)$ in sesamin group. The serum levels were similar to the liver, but in other tissue the desaturation index of $n-3$ fatty acids was not increased by sesamin (data not shown).

\section{DISCUSSION}

Shimizu et al. reported that sesamin was a potent and specific inhibitor of $\Delta 5$ desaturase of rat liver microsomes using $\left[{ }^{14} \mathrm{C}\right] 20: 3(n-6)(5)$. We previously reported that sesamin inhibited $\Delta 5$-desaturation of $n-6$ fatty acids but not $n-3$ fatty acids in rat hepatocytes (6). In this study, we investigated the effect of sesamin on $\Delta 5$-desaturation in vivo using rats fed $\gamma$-linolenic acid-rich or $\alpha$-linolenic acid-rich diets. Its effect on the metabolism of $n-6$ and $n-3$ fatty acids was compared, because only $\Delta 5$-desaturation of $n-6$ fatty acids has been investigated so far. 
Although we did not examine the absorption of sesamin, Hirose et al. reported that sesamin was detected by HPLC at concentrations of $0.17 \mu \mathrm{g} / \mathrm{ml}$ serum and 1.32 $\mu \mathrm{g} / \mathrm{g}$ liver when rats were fed the diet containing $0.5 \%(\mathrm{w} / \mathrm{w})$ of sesamin $(15)$.

In both Exps. 1 and 2, sesamin inhibited the $\Delta 5$-desaturation from $20: 3(n-6)$ to $20: 4(n-6)$ in the liver. However, the ratio of $20: 5(n-3)$ to $18: 3(n-3)$ in the liver suggested that sesamin did not inhibit $\Delta 5$-desaturation of $n$ - 3 fatty acids. These results are the same as those reported for rat hepatocytes (6). Although the ratio of $20: 5(n-3)$ to $20: 4(n-3)$ in kidney, heart, and lung was slightly decreased by sesamin feeding, the desaturation index of serum was similar to that of liver (data not shown). However, the findings in the liver are thought to be important because the liver is where most fatty acids are metabolized.

It is generally agreed that $n-3$ and $n-6$ fatty acids compete in the same enzyme systems (7-9). The desaturation steps in this pathway are considered given that the $n-3$ series fatty acids are preferable to $n-6$ series fatty acids (10). From these points of view, the competition of the metabolism of both series of PUFAs and the balance of intake of both types of fatty acids is still being discussed, but, in fact, the activity of desaturase on $n$ - 3 fatty acids has not been measured. Moreover, $\Delta 5$-desaturase has not been purified yet and the properties of the enzyme is not yet known. Christiansen et al. concluded that the desaturases for the $n-6$ and $n-3$ series of PUFAs are different based on the finding that the activities of desaturases in liver microsome of rats fed diets with different ratios of $n-6$ to $n-3$ were different when $18: 2(n-6)$ and $18: 3(n-3)$ were used as substrate (16). Our results support the possibility that metabolism of both types of PUFAs ( $n-6$ and $n-3) 45$-desaturase is different or that the affinity of $\Delta 5$-desaturase for both types of PUFAs is different.

In this study, we also observed that sesamin treatment significantly increased the rat liver weight. Sugano et al. $(2,15)$ reported the same results in rats fed diet containing sesamin and the increases of phospholipid. Huang et al. reported that 18 $: 3(n-6)$ and its metabolites competed with $n-3$ fatty acids, whereas $18: 4(n-3)$ metabolites competed also with $n-6$ fatty acids for incorporation into phospholipids (17). It is not yet known how sesamin inhibits the desaturation of $n-6$ and $n-3$ PUFAs after their incorporation into phospholipids. Sesamin inhibited the desaturation of $n-6$ fatty acids in PC more than PE. From these results, sesamin might affect the incorporation of the essential fatty acids into phospholipids. A study of competition between $n-6$ and $n-3$ fatty acids is needed to further clarify phospholipid metabolism.

We greatly acknowledge Dr. K. Akimoto, Institute for Biomedical Research, Suntory Ltd., Osaka, Japan, for the donation of sesamin. We also express our thanks to Idemitsu Petrochemical Co. Ltd., Tokyo, Japan, for the donation of $\gamma$-linolenate-rich mould oil.

\section{REFERENCES}

1) Shimizu, S., Akimoto, K., Kawashima, H., Shinmen, Y., and Yamada, H. (1989): 
Production of dihomo- $\gamma$-linolenic acid by Mortierella alpina 1S-4. J. Am. Oil Chem. Soc., 66, 237-241.

2) Shimizu, S., and Yamada, H. (1990): Production of dietary and pharmacologically important polyunsaturated fatty acids by microbiological process. Comments. Agric. Food Chem., 2, 211-235.

3) Akimoto, K., Yamada, H., Shinmen, Y., and Shimizu S. (1989): Production of bishomo-gamma-linolenic acid by fungi. Eur. Pat. Appl., pp. 14.

4) Sugano, M., Inoue, T., Koba, K., Yoshida, K., Hirose, N., Shinmen, Y., Akimoto, K., and Amachi, T. (1990): Influence of sesame lignans on various lipid parameters in rats. Agric. Biol. Chem., 54, 2669-2673.

5) Shimizu, S., Akimoto, K., Shinmen, Y., Kawashima, H., Sugano, M., and Yamada, H. (1991): Sesamin is a potent and specific inhibitor of $\Delta 5$ desaturase in polyunsaturated fatty acid biosynthesis. Lipids, 26, 512-516.

6) Fujiyama-Fujiwara, Y., Umeda, R., and Igarashi, O. (1992): Effect of sesamin and curcumin on 45 -desaturation and chain elongation of polyunsaturated fatty acid metabolism in primary cultured rat hepatocytes. J. Nutr. Sci. Vitaminol., 38, 353-363.

7) Mahfouz, M. M., and Kummerow, F. A. (1989): Effect of magnesium defficiency on $\Delta 6$ desaturase activity and fatty acid composition of rat liver microsomes. Lipids, 24, 727-732.

8) Mohrhauer, H., and Holman, R. T. (1963): Effect of linolenic acid upon the metabolism of linoleic acid. J. Nutr., 81, 67-74.

9) Rahm, J. J., and Holman, R. T. (1964): Effect of linoleic acid upon the metabolism of linolenic acid. J. Nutr., 84, 15-19.

10) Holman, R. T. (1964): Nutritional and metabolic interrelations among fatty acids. Fed. Proc., 23, 1062-1067.

11) Folch, J., Lees M., and Sloane-Stanley, G. H. (1957): A simple method for the isolation and purification of total lipids from animal tissues. J. Biol. Chem., 266, 497509.

12) Rao, G. A., Riley, D. E., and Lakin, E. C. (1985): Comparison of the thin layer chromatography/flame ionization detection system with other methods for the qantitative analysis of liver lipid contents in alcohol-fed rats and controls. Lipids, 20, 536541.

13) Fujiyama-Fujiwara, Y., Ohmori, C., and Igarashi, O. (1989): Metabolism of $\gamma$ linolenic acid in primary cultures of rat hepatocytes and in HepG2 cells. J. Nutr. Sci. Vitaminol., 35, 591-611.

14) Skipski, V. P., Smonlwe, A. F., Sullivan, R. C., and Barcley, M. (1965): Separation of lipid classes by thin-layer chromatography. Biochim. Biophys. Acta, 106, 386-896.

15) Hirose, N., Inoue, T., Nishihara, K., Sugano, M., Akimoto, K., Shinmen, S., and Yamada, H. (1991): Inhibition of cholesterol absorption and synthesis in rats by sesamin. J. Lipid Res., 32, 629-638.

16) Christiansen, E. N., Lund, J. S., Rørtveit, T., and Rustan, A. C. (1990): Effect of dietary $n-3$ and $n-6$ fatty acids on fatty acid desaturation in rat liver. Biochim. Biophys. Acta, 1082, 57-62.

17) Huang, Y.-S., Smith, R. S., Redden, P. R., and Cantrill, R. C. (1991): Modification of liver fatty acid metabolism in mice by $n-3$ and $n-6 \Delta^{6}$-desaturase substrates and products. Biochim. Biophys. Acta, 1082, 319-327. 